\title{
Multi-Stage PIC Receiver with decision thresholds over Rayleigh fading channel
}

\author{
Mohamed Moussaoui \\ Laboratoire Labtic \\ National School of applied Sciences \\ Tangier, Morocco \\ md.moussaoui@gmail.com
}

\author{
Mostafa Zaizouni \\ Alcatel-Lucent \\ Paris, France \\ mostafa.zaizouni@alcatel-lucent
}

\author{
Jean-Michel Rouvaen \\ IEMN-DOAE, University of \\ Valenciennes. \\ Valenciennes, France. \\ jean-michel.rouvaen@univ- \\ valenciennes.fr
}

\begin{abstract}
Parallel interference cancellation (PIC) is a family of low complexity multi-user detection methods for DS/CDMA systems. The performance of the multistage PIC depends on the decision function used in the interference cancellation. In this paper, we propose to provide the performance of PIC receiver using $\mathrm{Xha}$ function 'clipping' with respect to decision threshold value. The decision threshold determines how much MAI is to be cancelled with hard decision and high probability. Otherwise, we used a soft bit decision to cancel only the interference of unreliable users form the received signal. This approach is original compared to PIC receiver with decision thresholds using null zone hard decision. It is shown that the proposed detector performs well under realistic conditions, particularly in the case of error optimisation of decision threshold value.
\end{abstract}

Keywords-Code division multiple access, Multi-user detection, Parallel Interference Cancellation, Partial cancellation, Decision threshold

\section{INTRODUCTION}

Tn order to enhance capacity and near-far resistance of a DS-CDMA system, third generation systems may employ parallel interference cancellation (PIC) on the uplink. Previous work has shown that direct PIC results in a bias of the desired signal component, caused by the accumulation of small correlations between the desired signal and interference $[1,2,3]$. One technique for mitigating this bias is weighting of the estimated interference components by a SCF, prior to cancellation. This SCF is proportional to the confidence in the reliability of the interference estimate, and decreases at higher interference levels. Because the SCF is typically less than one, the resulting cancellation technique is often termed partial PIC [2, 3, 4]. Optimization of the SCF has been previously attempted in [2] and [3]. In [3], the authors derive a semi-analytic result for the SCF that minimizes BER of the desired user as a function of signal-to-noise ratio, interference level, and processing gain. In [2], the optimal SCF for an arbitrary stage of interference cancellation is computed as a function of system parameters and the reliability of the data estimate at the previous stage. For linear (soft) estimates of the symbol, it's shown that the approaches of [2] and [3] are equivalent. The linear estimate can be viewed as a joint estimate of the symbol and the channel assuming perfect channel knowledge.

For hard estimates of the symbol, this technique has been known as the PIC receiver using null zone hard decision.

In $[5,6]$ the performance of the PIC receiver using null zone hard decision in Rayleigh fading channel is provided with respect to the decision threshold value, and 'adequate' decision threshold value at each stage is obtained.

In this paper, we seek to combine the advantages of hard and soft decision functions with hybrid cancellation. When the signal amplitude estimation for each user is small compared to the decision threshold value, linear decision cancellation is used. Otherwise hard decision cancellation is employed, which will be correct with high probability. This decision function was proposed initially and analysed by Xha [7] in the context of multistage SIC with amplitude averaging.

We propose to provide the performance of PIC receiver using Xha function 'clipping' with respect to decision threshold value. Comparisons with PIC receiver using null zone hard decision in [5] show that the proposed detector performs well under realistic conditions, particularly in the case of error optimisation of decision threshold value.

\section{PARALLEL INTERFERNCE CANCELLATION}

We consider the base-station receiver for the asynchronous uplink CDMA channel with binary phase shift keying (BPSK) modulation. Let us consider $\mathrm{K}$ single-user direct sequence spread spectrum (DS-SS) transmitters, communicating simultaneously at the same rate over a linear additive white Gaussian noise (AWGN) channel. Once each user's binary data stream $b_{k}(t)$ is spread by its PN code waveform $c_{k}(t), N$ is the processing gain.

The resulting signal is:

$$
\mathrm{s}_{\mathrm{k}}(\mathrm{t})=\sqrt{\mathrm{P}_{\mathrm{k}}} \mathrm{b}_{\mathrm{k}}(\mathrm{t}) \mathrm{c}_{\mathrm{k}}(\mathrm{t}) \text {, }
$$

where $\mathrm{P}_{k}$ is the baseband power of the $\mathrm{k}^{\text {th }}$ user's signal.

It will be assumed, in all further analysis, that $b_{k}(t)$ and $c_{k}(t)$ are statistically independent random processes. The 
received signal $r(t)$ is expressed as:

$$
\mathrm{r}(\mathrm{t})=\sum_{\mathrm{k}=1}^{\mathrm{K}} \mathrm{S}_{\mathrm{k}}\left(\mathrm{t}-\tau_{\mathrm{k}}\right)+\mathrm{n}(\mathrm{t})
$$

Where $\tau_{k}$ the delay for the kth user and the noise is $n(t)$ is modelled as zero-mean ergodic complex AWGN process with one-sided power spectrum magnitude $\mathrm{N}_{0}$.

Consider a PIC receiver Having $\mathrm{S}$ stage (see figure 1). The first stage provides first order amplitude estimates for each user, the second stage then constructs MAI signal estimates and cancels this reconstructed MAI from the original received signal. Interference cancellation can be repeated at each stage. In the branch the kth user in the sth stage, $1 \leq_{\mathrm{S}} \leq \mathrm{S}$, the regenerated interference signals $\left(\hat{\mathrm{s}}_{\mathrm{j} \neq \mathrm{k}}^{(\mathrm{s})}(\mathrm{t})\right)$ form users other than the kth user are cancelled from the received signal. After interference cancellation, the signal for the kth user in the sth stage is given by

$$
\mathrm{r}_{\mathrm{k}}^{(\mathrm{s})}(\mathrm{t})=\mathrm{r}(\mathrm{t})-\sum_{\substack{\mathrm{j}=1 \\ \mathrm{j} \neq \mathrm{k}}}^{\mathrm{K}} \hat{\mathrm{s}}_{\mathrm{k}, \mathrm{j}}^{(\mathrm{s})}(\mathrm{t})
$$

The user k's sth stage metric for ith bit is computed according to:

$$
\mathrm{z}_{\mathrm{k}, \mathrm{i}}^{(\mathrm{s})}=\frac{1}{\mathrm{~T}} \int_{\mathrm{i} \psi_{+} \tau_{\mathrm{k}}}^{(\mathrm{i}+1) \mathrm{T}+\tau_{\mathrm{k}}} \hat{\mathrm{r}}_{\mathrm{k}}^{(\mathrm{s})}(\mathrm{t}) \mathrm{c}_{\mathrm{k}}\left(\mathrm{t}-\mathrm{i} \mathrm{T}-\tau_{\mathrm{k}}\right) \mathrm{dt} .
$$

The output of the receiver for the ith bit of the kth user is weighted by the tentative decision function: $\hat{\mathrm{b}}_{\mathrm{k}, \mathrm{i}}^{(\mathrm{s})}=\mathrm{f}\left(\mathrm{z}_{\mathrm{k}, \mathrm{i}}^{(\mathrm{s})}\right)$

\section{MULTI-STAGE PARTIAL PIC RECEIVER WITH DESCISION THRESHOLD}

The multi-stage PIC receiver causes a bias in decision statistic at the output of the MF bank [1, 2, 3].

To mitigate the effect of the bias, the multi-stage PIC receiver with partial cancellation is used.

There are two types of multi-stage partial PIC receivers: the multistage PIC receiver with partial cancellation factors and multistage PIC receiver with decision thresholds.

In the multi-stage PIC receiver with partial cancellation factors, the MAI estimate is multiplied by a partial cancellation factor between 0 and 1 , and then subtracted from the received signal in each stage of the receiver.

In the multi-stage PIC receiver, the subtraction of an interfering signal based on an incorrect symbol decision may significantly increase interfering power from the signal. To mitigate this problem, the multi-stage PIC receiver with decision thresholds is used.

The PIC receiver performs partial cancellation by using the null zone function is defined by:

$$
\widehat{b}^{(s)} \equiv\left\{\begin{array}{l}
1, \quad z^{(s)}>\lambda^{(s)} \sqrt{E_{b}}, \\
0,\left|z^{(s)}\right| \leq \lambda^{(s)} \sqrt{E_{b}}, \\
-1, \quad z^{(s)}<-\lambda^{(s)} \sqrt{E_{b}}
\end{array}\right.
$$

where $T h^{(s)}=\lambda^{(s)} \cdot \sqrt{E_{b}}$ is a decision threshold and $\lambda^{(s)}$ is a parameter which adjusts the decision threshold in the sth stage.

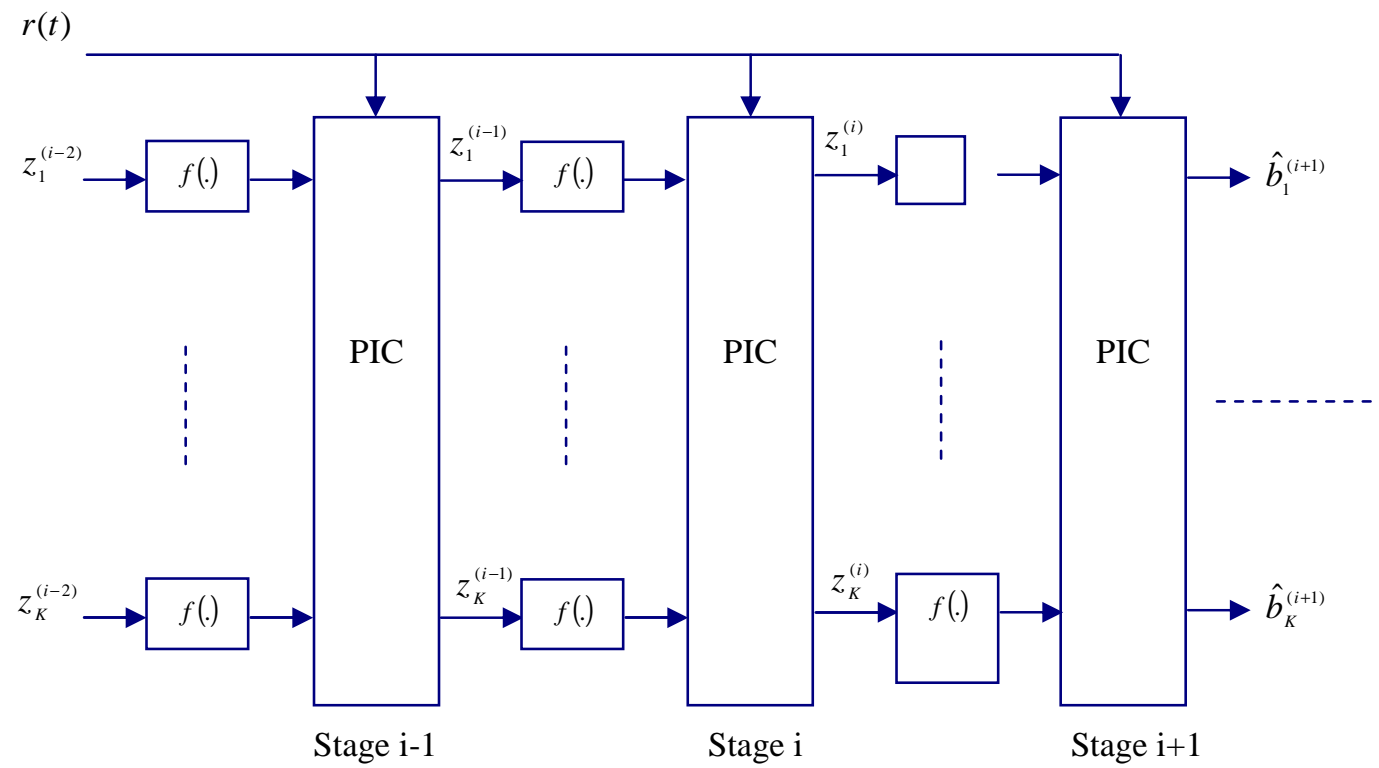

Fig. 1 Multistage Parallel interference cancellation Receiver 


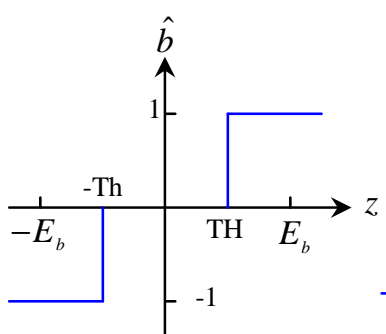

(a) Null-zone

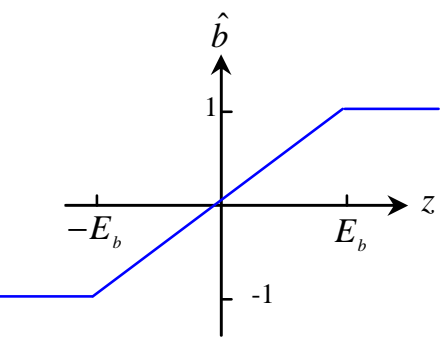

(b) Unit-clipper

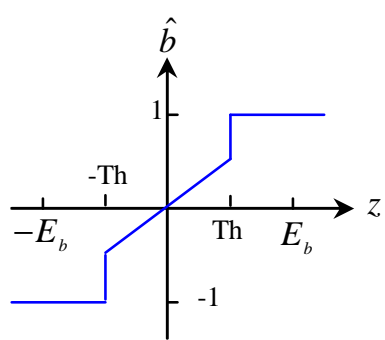

(c) Xha decision function : clipping

Fig. 2 Decision functions. (a) null zone . (b) Unit-clipper. (c) Xha decision function: clipping

The null zone function (figure 2-a) only uses symbols in the interference calculation, if their absolute value is higher than the threshold.

The decision threshold determines the amount of MAI to be cancelled in partial cancellation. In [5], a new PIC receiver with an adaptive decision threshold is proposed. The proposed PIC receiver is the improved version of the PIC receiver using null zone hard decision.

The decision threshold for the ith bit in the sth stage is given by

$T h_{i}^{(s)}=p^{(s)} \cdot\left(\frac{\sigma_{i}^{(s)}}{\sqrt{E_{b}}}\right)^{n^{(s)}} \cdot \sqrt{E_{b}}$

where $n^{(s)}$ and $p^{(s)}$ are parameters which adjust the decision threshold in the sth stage and $\sigma_{i}^{(s)}$ is the sample standard deviation in the sth stage for the ith bit time.

This decision threshold is the generalized form and the decision threshold for null zone hard decisions is one of the special forms with $n^{(s)}=0$.

The linear decision function offers analytical access to the PIC performance, but performs worse than other decision functions. The limiter in the unit-clipper decision function (figure 2-b) improves performance over the linear PIC. However, the unit-clipper cancels only the part of the noise above the amplitude limit.

In [7], Xha propose and analyze a family of generalized unit-clipper bit decision functions that better combine linear and hard decisions in the context of multistage SIC.

We propose the Xha decision function 'clipping' (figure 2c) to be used in the multistage PIC receiver, and analyze its steady-state performance.

In the $s$-th stage of the receiver, the $i$-th symbol estimate for the user $k$ is given by

$$
\widehat{b}^{(s)} \equiv\left\{\begin{array}{l}
1, \quad z^{(s)}>T h^{(s)}, \\
z^{s},\left|z^{(s)}\right| \leq T h^{(s)}, \\
-1, \quad z^{(s)}<-T h^{(s)}
\end{array}\right.
$$

Where $T h^{(s)}$ is the threshold at stage s.

The decision threshold determines how much MAI is to be cancelled with hard decision and high probability.

Otherwise, we use a soft bit decision to cancel only the interference of unreliable users form the received signal. This approach is original compared to PIC receiver with decision thresholds using null zone hard decision.

In each stage of the multi-stage PIC receiver using null zone hard decision, as a user's signal is not reliable when its signal to interference ratio (SIR) is small, it is better not to attempt to cancel the interference from that signal.

The fact that used soft estimated interference in the processes of cancellation increase the performance of PIC receiver compared to the null zone hard decision.

The decision threshold in the $s$-th stage is given by $T h^{(s)}=\lambda^{(s)} \sqrt{E_{b}}$, where $\lambda^{(s)}$ is the parameter which adjusts the decision threshold in the $s$-th stage. The optimum values of $\lambda^{(s)}$ to achieve the lowest BER are found by trialand-error with the restriction that

$\lambda^{(1)}>\lambda^{(2)}>$. . . $>\lambda^{(s)}>0$.

The users are divided into two groups: reliable and unreliable users.

The proposed PIC receiver used the null zone decision to cancels the interference from reliable users, and clipping decision form unreliable users.

After interference cancellation, the signal for the k-th user in the s-th stage is given by

$r_{k}^{(s)}(t)=r(t)-\sum_{\substack{j=1 \\ j \neq k \in R}}^{K} \hat{x}_{j}^{(s)}(t)-\sum_{\substack{i=1 \\ i \neq k \in \Gamma}}^{K} \tilde{x}_{i}^{(s)}$,

where $\mathrm{R}$ is the set of indices of reliable users, and $\Gamma$ is the set of indices of unreliable users.

\section{SIMULATION RESULTS}

In this section we investigate the effectiveness of the proposed PIC receiver with clipping decision function through computer simulations.

In the following simulations, an asynchronous DS-CDMA system was considered with $\mathrm{K}$ users. BPSK modulation is assumed. A random signature sequence is assigned to each user and the processing gain $L$ is 31 . Assume that the channel for each user has independent identically distributed frequency-flat Rayleigh fading. Also assume that the receiver knows the signature sequences of all users and has perfect synchronization and channel estimation for each user. 

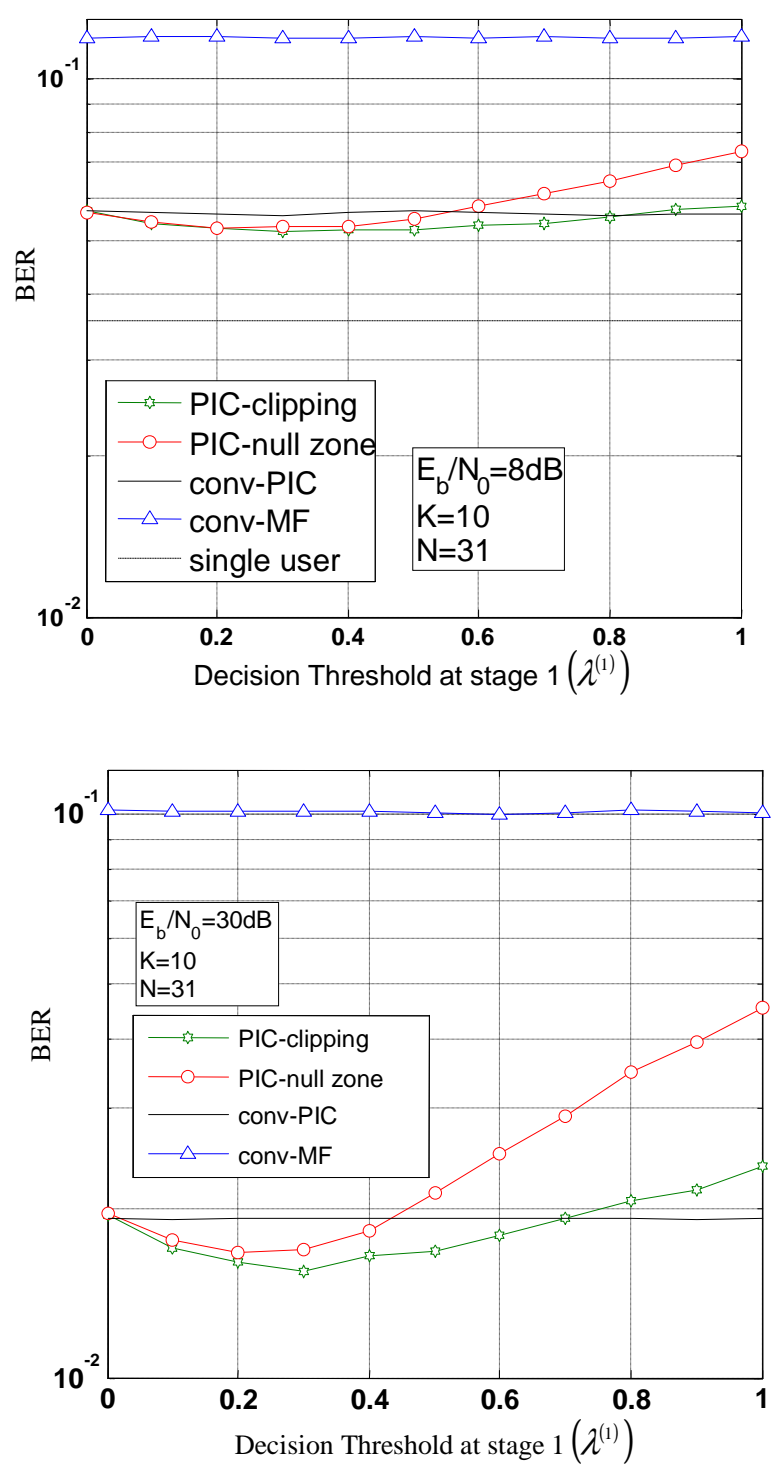

Fig. 3 BER versus $\lambda^{(1)}, S=1, K=10, N=31$.

Figure 3 shows the simulated BERs versus $\lambda^{(1)}$ in a Rayleigh fading channel for the number of users $\mathrm{K}=10$ and the number of stages $S=1$. For PIC receiver using null zone hard decision, it is shown that the lowest BER can be obtained with the parameter $\lambda^{(1)}=0.2$ for both case $E_{b} / N_{0}=8 d B$ and $E_{b} / N_{0}=30 d B$.

For PIC receiver using clipping decision, it is shown that the lowest BER can be obtained with the parameter $\lambda^{(1)}=0.2$ for $E_{b} / N_{0}=8 d B$, and $\lambda^{(1)}=0.3$ for $E_{b} / N_{0}=30 d B$.

Figure 4 shows the simulated BERs versus $\lambda^{(3)}$ in a Rayleigh fading channel for the number of users $K=10$, the number of stages $\mathrm{S}=3$.

For null zone hard decision, it is shown that the lowest BER can be obtained with the parameters $\lambda^{(1)}=0.6, \lambda^{(2)}=0.3$ and $\lambda^{(3)}=0.2$ for $E_{b} / N_{0}=8 d B$.
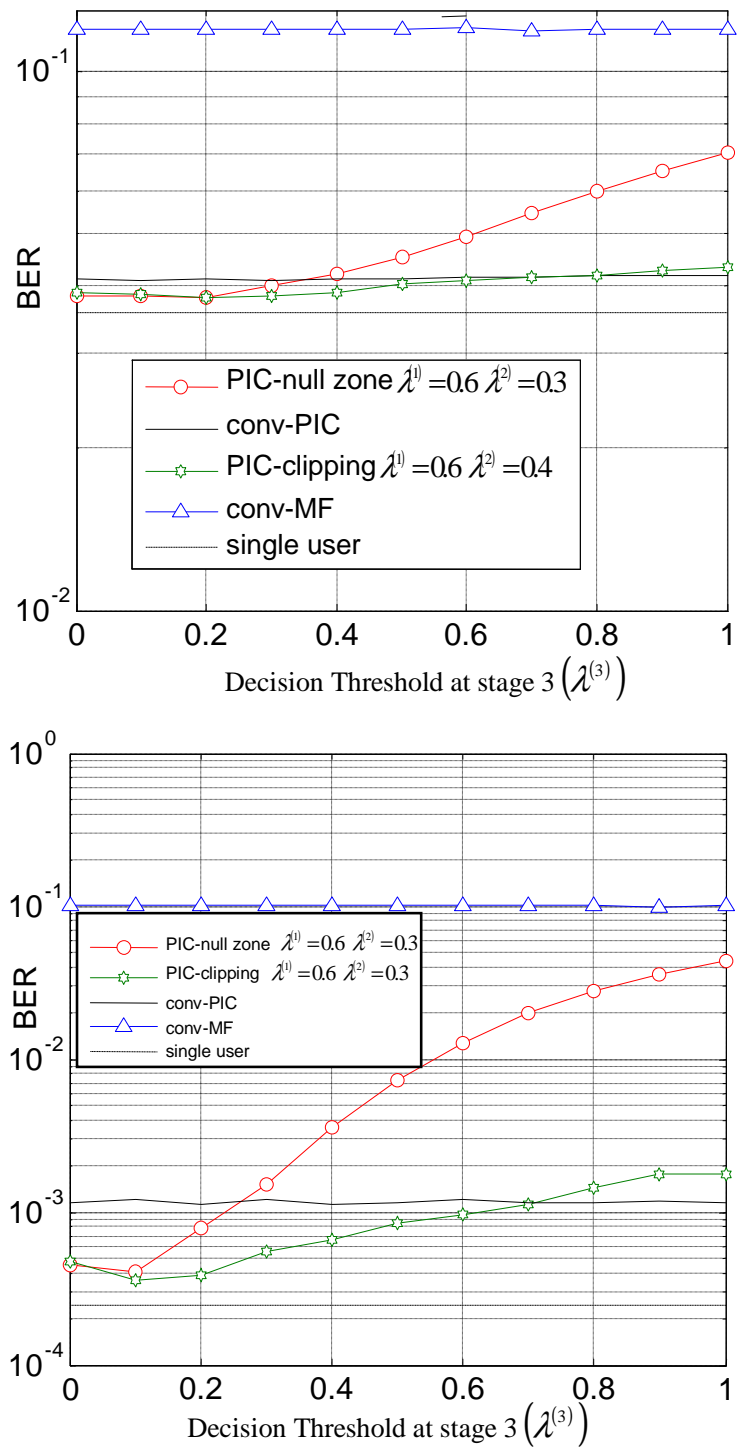

Fig. 4 BER versus $\lambda^{(3)}, S=1, K=10, N=31$.

For $E_{b} / N_{0}=30 d B$, the optimal parameters are $\lambda^{(1)}=0.6$, $\lambda^{(2)}=0.3$ and $\lambda^{(3)}=0.1$.

For clipping decision, it is shown that the lowest BER can be obtained with the parameters $\lambda^{(1)}=0.6, \lambda^{(2)}=0.4$ and $\lambda^{(3)}=0.2$ for $E_{b} / N_{0}=8 d B$.

For $E_{b} / N_{0}=30 d B$, the optimal parameters are $\lambda^{(1)}=0.6$, $\lambda^{(2)}=0.3$ and $\lambda^{(3)}=0.1$.

The reason why we selected the parameters of the previous stages is that from the simulation results and trial-and-error search the optimal BER performance is obtained when we set the parameter as above.

It is shown that the BER performance of the proposed PIC receiver is better than those of the conventional PIC receiver and the PIC receiver using null zone hard decision for all stage. 
The PIC receiver using null zone hard decision is very sensitive to variations in threshold, and its performance deteriorates rapidly according to error optimization of decision threshold value.

For all figure, our proposed detector shows a high degree of resistance to error optimization of decision threshold value At stage 3 and $E_{b} / N_{0}=8 d B$, its shown that PIC receiver using null zone decision and conventional PIC receiver achieve similar BER performance at error of optimization $\Delta \lambda^{(3)} \approx 0.1$.

In the same condition, our proposed detector achieves similar performance at error of optimisation $\Delta \lambda^{(3)}=0.5$.

\section{CONCLUSION}

In this paper, the performance of the PIC receiver using 'clipping' decision is shown for the reverse link of DSCDMA system in Rayleigh fading channel as we vary the decision threshold at each stage.

Our proposed detector uses a soft bit decision to cancel only the interference of unreliable users form the received signal. It is shown that our proposed detector achieve significant performance improvement over the PIC receiver with null zone hard decision in each stage, particularly in the case of error optimization of decision threshold value.

\section{REFERENCES}

[1] M. Moussaoui, M. Zaizouni , J. M. Rouvaen, “Another approach for partial parallel interference cancellation," Wireless Personal Communications, Volume 42, Number 4, September 2007, pp. 587606(20).

[2] D. Divsalar, M. Simon, D. Raphaeli, "Improved parallel interference cancellation for CDMA," IEEE Trans. Commun., vol. 46, pages 258268, Février 1998.

[3] P.G. Rennuci, B.D. Woerner, "Optimization of soft interference cancellation for DS-CDMA," IEE Electronics Letters, vol. 34, pp. 731.733, Avril 1998 .

[4] Sunmi Kim, Jaewon Park, Yongwan Park, "MIMO partial parallel interference cancellation for space time block coded MIMO-CDMA system," The 8th International Conference on Advanced Communication Technology, 2006. ICACT 2006. Volume 2, Issue , 20-22 Feb.

[5] B.Y. Cho Jae, J.H. Lee, "Nonlinear parallel interference cancellation with partial cancellation for a DS-CDMA system," IEICE Trans. Commun., vol. E83-B, No. 9, pages 2141-2146, Septembre 2000.

[6] S. H. Han and J. H. Lee, "Multi-stage partial parallel interference cancellation receivers for multi-rate DSCDMA system," IEICE Trans. Commun., vol. E86-B, no. 1, pp. 122-132, Jan. 2003.

[7] W. Zha, S.D. Blostein, "Soft-Decision Multistage Multiuser Interference Cancellation," IEEE Transactions on Vehicular Technology, Vol. 52, No. 2, pages 380-389, Mars 2003. 